Paper ID \#20368

\title{
Algae for STEM Education
}

\section{Dr. Kauser Jahan, Rowan University}

Kauser Jahan, is a Professor of Civil and Environmental Engineering at Rowan University. She received her B.S.C.E. from the Bangladesh University of Engineering and Technology, an MSCE from the University of Arkansas, Fayetteville and a Ph.D. from the University of Minnesota, Minneapolis. Her passion as an educator and mentor has been recognized by many professional organizations over the years. She is the recipient of the Gloucester County Women of Achievement Award, Lindback Foundation Teaching Award, the NJ ASCE Educator of the Year award, the Gary J. Hunter Excellence in Mentoring Award, the ASEE Environmental Engineering Division Meritorious Service Award, the ASEE Women in Engineering Division Sharon A. Keillor Award and the WEPAN Women in Engineering Initiative Award. She has been instrumental in establishing the Attracting Women into Engineering, the Engineers on Wheels and Engineering Clinics for Teachers programs at Rowan University. She has served as the Institutional Representative and Advisory Board Chair for the Women's Professional Network at Rowan University for six years and currently is an advisory board member of the New Jersey Chapter of the American Council on Education (ACE) Office of Women in Higher Education (OWHE). She received a Fulbright award in 2015.

\section{Dr. Stephanie Farrell, Rowan University}

Dr. Stephanie Farrell is Professor and Founding Chair of Experiential Engineering Education at Rowan University (USA) and was 2014-15 Fulbright Scholar in Engineering Education at Dublin Institute of Technology (Ireland). From 1998-2016, Stephanie was a faculty member in Chemical Engineering at Rowan. Dr. Farrell has contributed to engineering education through her work in experiential learning, focusing on areas of pharmaceutical, biomedical and food engineering. She has been honored by the American Society of Engineering Education with several teaching awards such as the 2004 National Outstanding Teaching Medal and the 2005 Quinn Award for experiential learning.

\section{Dr. Ying Tang, Rowan University}

Ying Tang received the B.S. and M.S. degrees from the Northeastern University, P. R. China, in 1996 and 1998, respectively, and Ph.D degree from New Jersey Institute of Technology, Newark, NJ, in 2001. She is currently a Professor of Electrical and Computer Engineering (ECE) at Rowan University, Glassboro, NJ. Her research interests include virtual reality and augmented reality, artificial intelligence, and modeling and scheduling of computer-integrated systems. Dr. Tang is very active in adapting and developing pedagogical methods and materials to enhance engineering education. Her most recent educational research includes the collaboration with Tennessee State University and local high schools to infuse cyberinfrastructure learning experience into the pre-engineering and technology-based classrooms, the collaboration with community colleges to develop interactive games in empowering students with engineering literacy and problem-solving, the integration of system-on-chip concepts across two year Engineering Science and four year ECE curricula, and the implementation of an educational innovation that demonstrates science and engineering principles using an aquarium. Her work has resulted in over 100 journal and conference papers and book chapters.

\section{Dr. Cheryl A Bodnar, Rowan University}

Cheryl A. Bodnar, Ph.D., CTDP is an Assistant Professor in the Department of Experiential Engineering Education at Rowan University. Dr. Bodnar's research interests relate to the incorporation of active learning techniques in undergraduate classes as well as integration of innovation and entrepreneurship into the engineering curriculum. In particular, she is interested in the impact that these tools can have on student perception of the classroom environment, motivation and learning outcomes. She obtained her certification as a Training and Development Professional (CTDP) from the Canadian Society for Training and Development (CSTD) in 2010, providing her with a solid background in instructional design, facilitation 
and evaluation. She was selected to participate in the National Academy of Engineering (NAE) Frontiers of Engineering Education Symposium in 2013 and awarded the American Society for Engineering Education Educational Research Methods Faculty Apprentice Award in 2014.

\section{Dr. C. Stewart Slater, Rowan University}

C. Stewart Slater is a professor of chemical engineering and founding chair of the Chemical Engineering Department at Rowan University. He has an extensive research and teaching background in separation process technology with a particular focus on membrane separation process research, development and design for green engineering, and pharmaceutical and consumer products. He received his Ph.D., M.S., and B.S.in chemical and biochemical engineering from Rutgers University. Prior to joining Rowan University he was a professor at Manhattan College.

Dr. Mariano Javier Savelski, Rowan University

Professor and Chair of Chemical Engineering

\section{Dr. Parth Bhavsar, Rowan University}

Parth Bhavsar, is an assistant professor in the Department of Civil and Environmental Engineering at Rowan University. His research interests include Intelligent Transportation System (ITS), Connected, Autonomous, and connected-automated Vehicle Technologies, Transportation Data Analytics, and Alternative Fuel Vehicles. Dr. Bhavsar has published in peer reviewed journals such as the Transportation Research Part C: Emerging Technology, Transportation Research Part D: Transport and the Environment and Transportation Research Record Journal of the Transportation Research Board. Dr. Bhavsar was previously a postdoctoral fellow in a connected vehicle research program in the Glenn Department of Civil Engineering at Clemson University, where he worked on several connected vehicle technology research projects. Dr. Bhavsar received his Ph.D. degree in 2013 and his M.S. degree in 2006 from Clemson University. His Ph.D. dissertation focused on development of an evaluation framework for connected vehicle technology supported alternative fuel vehicles. Dr. Bhavsar also has three years of experience in the private sector in developing transportation engineering and planning solutions, specifically traffic micro-simulation projects.

\section{Angela D Wenger, Center for Aquatic Sciences}

Angela Wenger has worked in informal science education since 1991. She has been involved in many facets of the museum experience, including designing and presenting many of the museum's public programs and exhibits, youth development programs, programs for underserved audiences, and professional development experiences. Her background includes 23 years of scientific research in a myriad of science topics, as well as psychology and family learning in museums. She has taught general biology, chemistry, aquatic science, and ecology for 20 years.

Ms. Wenger is active in a variety of professional informal education organizations and is co-founder and chairperson of the Mid-Atlantic YouthALIVE! Regional Network. She has co-authored two publications focused on family learning. Ms. Wenger is deeply commitment to diversity issues and broadening access to science for underserved audiences. She is also passionate about professional development of youth and staff working in science centers and museums.

Miss Patricia Lynn Hurley, Rowan University

Ms. Roisin Breen, Rowan University

Dr. DeMond S Miller, Department of Sociology and Anthropology

DeMond S. Miller is a Professor of Sociology and Director of The Program in Disaster Preparedness and Emergency Management.

\section{Dr. Kara Ieva Ieva, Rowan University}


Dr. Kara Ieva is currently an Associate Professor in the Counseling in Educational Settings program at Rowan University. Kara's areas of research interest include counseling children and adolescent of underserved populations regarding college and career readiness particularly in STEM and group counseling. Further, she was the principal investigator and project director for the Rowan University Aim High Science and Technology Academies (grant funded; \$998,259), that aids first generation and low-income college students access and preparation for post-secondary education in STEM. She currently serves a Co-Pi on a grant from the Forman S. Acton Foundation $(\$ 198,000)$ to evaluate funded college access programs.

\section{Megan Casey Mittenzwei}




\title{
Algae Grows the Future
}

\begin{abstract}
In 2008, a list of engineering challenges put forth by the National Academy of Engineering (NAE) identified the need for technological innovations for global progress. These challenges focused on education, energy, infrastructure, clean water, climate change, and much more. In an effort to help foster a generation of engineers that are more aware of their role in making the world a better place, a partnership was formed with the Adventure Aquarium Center for Aquatic Sciences (CAS). Funded by the National Science Foundation, the goal of this partnership was to form a multidisciplinary engineering team focused on adapting current experiments to more environmentally conscious alternatives. These experiments have begun to be implemented in freshman engineering courses, the STEM Academy at Glassboro High School, and K-12 outreach programs. Ultimately, the purpose of these experiments is to reduce environmental impact by replacing current environmental processes with algae based alternatives. By partnering with the CAS, the University gained access to its algae production facility, a powerful tool in researching the many uses of algae. Algae-based experiments and hands-on activities in the fields of water resources engineering, environmental engineering, cosmetics, and nutrition have been developed. By focusing on these fields, the positive impact that algae can have on issues faced by developing nations around the world was highlighted. Gas transfer and coagulation flocculation experiments have been used to research the sustainability of algae use in the water treatment process, with a focus on the feasibility of replacing current processes with algae based alternatives. Calorimeter tests have been conducted to research the potential nutritional benefit of algae based products. Exposure to social and environmental injustices along with ethics case studies are also an integral part of the project. Ultimately, the purpose of this project is to teach a generation of future engineers the impact that algae can have on solving humanitarian issues around the world.
\end{abstract}

\section{Introduction}

\subsection{Project Goals/Partnership}

Algae Grows the Future is a project focused on advancing a generation of engineers dedicated to improving the world for all people. Through the use of science and engineering, this project hopes to spark students' interest in engineering by redefining how discovery, learning, and innovation is approached in the classroom. The goals of the project are to ensure the highest quality of STEM education in order to improve learning and comprehension of engineering concepts. Additionally, the designed curriculum takes a multidisciplinary approach to teaching engineering in order to show how the different branches are connected and how to integrate critical thinking skills towards solving global issues. More specifically though, this project will show the benefit that algae based solutions can have on these global issues.

Algae Grows the Future was designed to be an innovative initiative with CAS at the Adventure Aquarium. As an organization that focuses on promoting the understanding, appreciation, and protection of aquatic life and habitats, through research, education, and youth development, CAS was the perfect fit for a project using aquatic life to teach the importance of education, research, and youth development. Furthermore, this partnership allows us to reach the over 50,000 
students and educators that CAS works with, extending the reach of our influence further than just our own university and local high schools.

\subsection{NAE Grand Challenges}

Leading technological thinkers from around the world were asked by the National Academy of Engineering to identify grand challenges for engineers in the 21 st century based on the world's technological advances and the current issues at hand all over the planet. These fourteen challenges have the intention of bettering the world by overcoming obstacles that will improve the quality of life not only for humans, but the environment and ecosystems that live alongside us. The NAE Grand Challenges include advancing personal learning, enhancing virtual reality, engineering better medicine, providing access to clean water, managing the nitrogen cycle, developing carbon sequestration methods, and engineering the tools of scientific discovery ${ }^{1}$. The Algae Grows the Future project addresses as many challenges as possible through an experiment based learning curriculum as well as the resulting applications of the experiments in research. The project's intention is to get students interested in global issues and provide a baseline knowledge of how alternative thinking can be adapted to develop solutions through the innovative application of experimental findings.

\subsection{Why Algae}

Algae are photosynthetic organisms that belong to a large and diverse group that is versatile in size and color. Many species are relatively inexpensive and easy to grow, making them useful at almost any level of education. The applications of algae, more importantly, are widely versatile, ranging from the cosmetics industry to biofuels. Some of the many known applications include nutrition, water treatment, medicine, fuel sources, pollution control, fertilizer, etc. Because algae are photosynthetic, they are fast growing and require little materials to maintain, making them an easily renewable resource. ${ }^{2}$ For the purpose of the curriculum developed at the University, the species chlorella vulgaris was used in all experiments and grown in large quantities for the use of the freshman engineering clinic program, other local schools, and even other projects within the department such as oil extraction in chemical engineering.
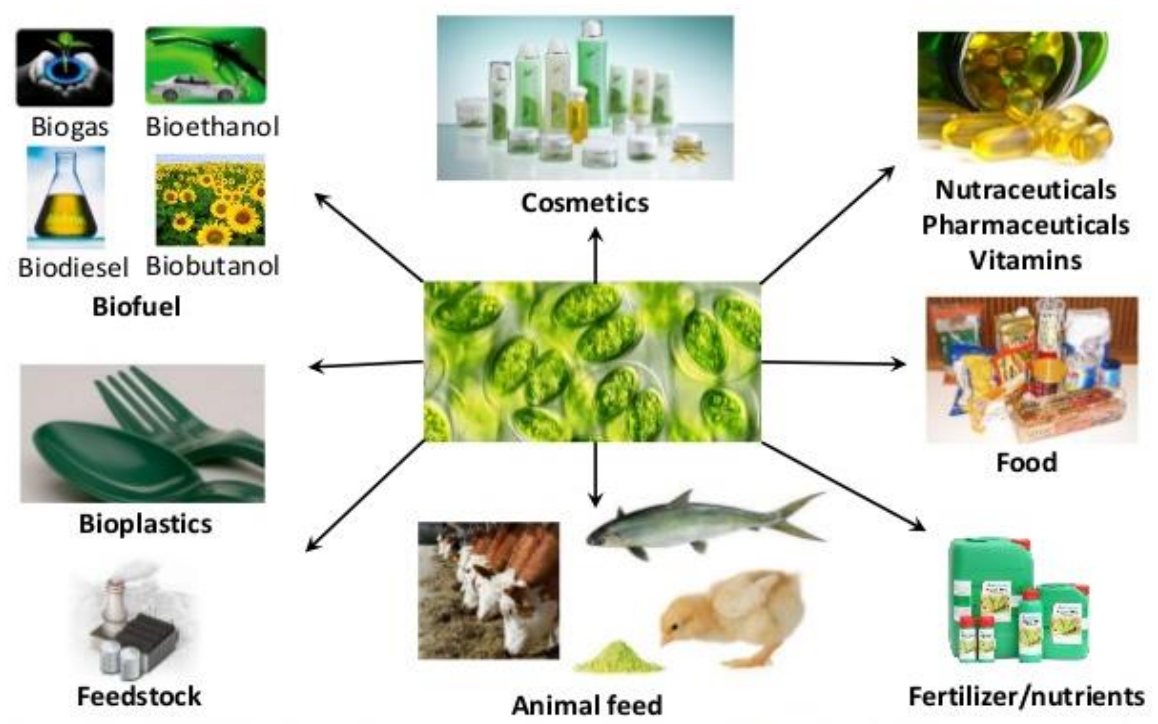

Figure 1: Uses of algae [8] 
Algae is a versatile and effective learning tool that poses a solution to some of the most challenging problems of today's world. Experiments using algae are multidisciplinary and incorporate a wide range of engineering concepts, but also ideas from the natural sciences and humanities. The basic knowledge behind algae innovations can be shown through algae samples grown anywhere, including college labs and K-12 classrooms.

\section{Curriculum}

\subsection{Global Engineers/Global Issues}

Engineering provides a unique platform to make an impact on the world. Engineers are responsible for utilizing the scientific principles studied in laboratories, patterns and laws derived by mathematicians, and innovative problem-solving to develop new products and processes or improve upon existing ones. These products and processes have an impact on consumers and the environment that surrounds them, both small and large. Thus, it is vital that engineers have a global perspective. While it is important to consider economics and efficiency, the scope of engineering extends beyond cost and industry. Engineers must recognize the effects that their projects will have socially, environmentally, and humanitarianly. Furthermore, it is important for the field of engineering to address global issues, including world hunger, dependency on fossil fuels, and limited access to proper healthcare, global warming, and much more.

In creating this curriculum, there was high emphasis on promoting global consciousness among students. The experiments and activities were designed not only to get students enthused about math, science, and engineering and teach them basic principles of experimentation, but also to encourage the consideration of public policy, social issues such as injustices, and ethics case studies.

This educational approach is becoming increasingly more important. In today's world, there are a lot of issues that need to be addressed, including global warming, world hunger, energy, and more. These issues are not going away anytime soon and it is important that the next generation of thinkers are prepared and excited to tackle them.

\section{Experiments}

\subsection{Calorimetry}

The prospect of algae as a nutritional food source and its potential application in the battle against world hunger is explored in a calorimetry experiment. Calorimetry involves the determination of energy change in a system through the measurement of heat exchange between the system and its surroundings. In terms of food science, calorimetry can be used to measure the amount of energy required to burn a food, often expressed as the number of Calories.

Students conduct a calorimetry experiment utilizing an empty, clean soda can as a calorimeter. The soda can is secured on a ring stand and filled with water of a measured initial temperature. A food sample is lit on fire with a match and placed beneath the soda can. The final water temperature is measured after the food sample is completely burned. The energy transferred from the burning food to the water is equal to the mass of water multiplied by the specific heat of water multiplied by the change in temperature. The energy calculated is the energy of the food, often referred to as Calories. 
In this experiment, students determine the number of calories in several foods, at least one of these foods being algae-based. The students are then able to compare the energy content of the food samples, along with their nutritional values, and analyze the nutritional benefits of algae.

According to the World Hunger Programme, about 795 million people in the world do not have the proper nutrition to live a healthy and active lifestyle. This is one out of every nine people. Furthermore, poor nutrition claims the lives of about 3.1 million children each year. ${ }^{3}$ The effects of malnutrition are widespread and Studies have shown that the best diet for malnutrition recovery consists of high protein and high fat intake. Algae is a food source that contains high protein and fat and therefore has potential as a future global food source.

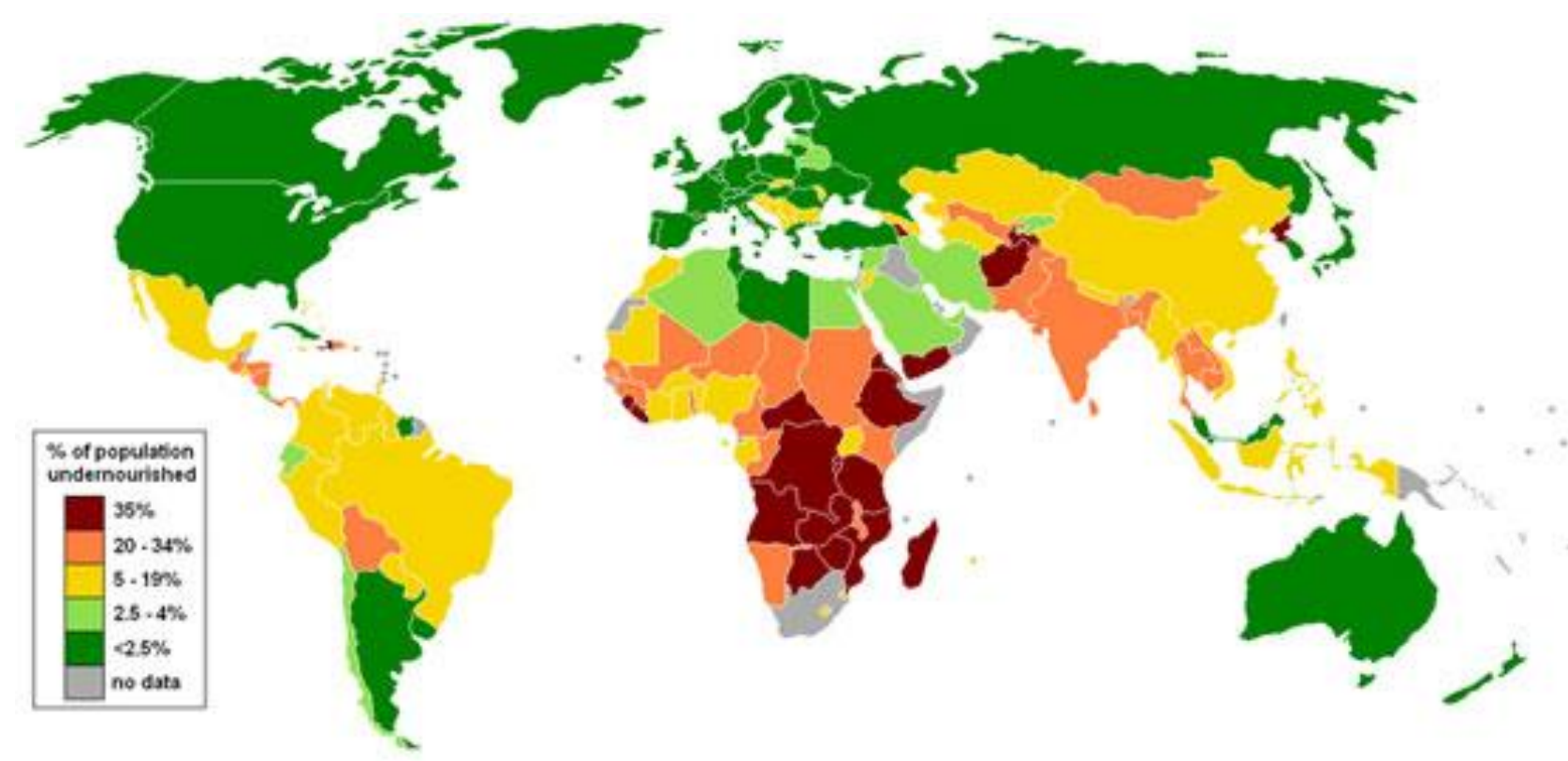

Figure 2: Percent population of undernourished by country [9]

\subsection{Cosmetics}

The components and properties of algae make it a beneficial ingredient in the cosmetic industry. Some products that utilize algae are algal soaps, algae anti-aging creams, algae beauty serums. The beneficial properties that make algae so appealing to the industry include its Omega3 fatty acid content, ability to regulate sebum and collagen production, and its richness in essential vitamins and minerals. These properties contribute to more hydrated, firmer skin and treatment of acne, wrinkling, and eczema. ${ }^{4}$

A study published in Women's Health Journal in 2011 concluded that mineral oil hydrocarbons are one of the leading contaminants to the human body. ${ }^{5}$ These hydrocarbons are often present in lotions and other cosmetic products, along with petroleum and questionable chemicals. These ingredients are lathered on and absorbed by the largest organ of the human body - the skin. Thus, consumers are at risk. By finding substitutes for these questionable ingredients, healthier products can be created and the usage of nonrenewable resources can be reduced.

Students are prompted to consider the ingredients in everyday items and see an application of algae first-hand through the creation of a personalized lip gloss. Students learn the basic 
components of lip gloss, which are oil and butter for moisture, wax for structure, and coloring and scent for personalization. Then, students make their own lip gloss using algae oil, beeswax, honey, coloring, and essential oil for scent.

\subsection{Photosynthesis}

The photosynthesis process serves as the fundamental step in understanding how algae can be used to solve global issues. In this simple experiment, algae is prepared ahead of time within a photosynthesis tank (or a beaker) that allows a dissolved oxygen meter to fit into the tank. Students first record the initial dissolved oxygen level within the tank. After, they record changes in the dissolved oxygen level with an added light source every minute for about fifteen minutes. Finally, they use the same procedure with a dark cover over the tank. From this experiment, students gain an understanding that the dissolved oxygen levels increase when the tank is exposed to the light source, and decrease when it is covered. This concept is evidence of the photosynthetic reaction in which carbon monoxide is used up to produce oxygen.

One of the 14 Grand Challenges that best aligns with this experiment is to "Develop Carbon Sequestration Methods." Photosynthesis is a recognized carbon dioxide sequestration mechanism. This experiment can help students understand the process of carbon sequestration through critical thinking skills. Algae is a preferred specimen as compared to traditional plants for many reasons, such as it's highly efficient photosynthesis process. ${ }^{6}$ The overall goal of this experiment is to expose students to the idea that algae can be used to solve some of the world's most challenging environmental problems.

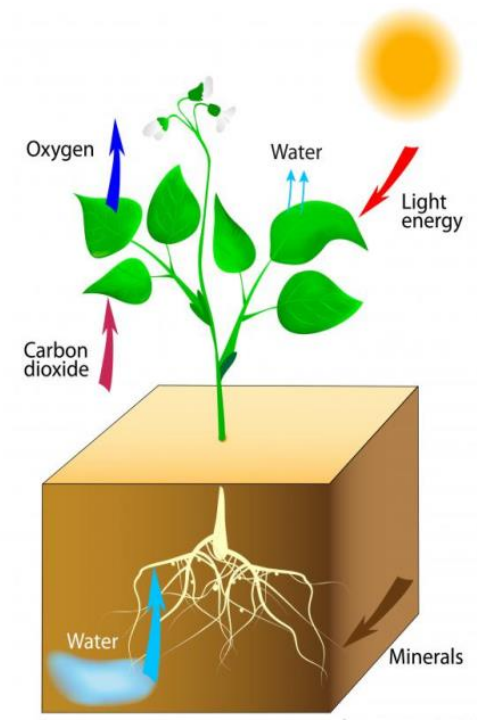

Figure 3: Process of carbon sequestration through photosynthesis [10]

\subsection{Effect of Light Intensity on Oxygen Production}

Similar to the photosynthesis lab described above, this experiment details how the photosynthetic nature of algae can be used to convert carbon dioxide into oxygen. Where this experiment differs though, is its focus on the relationship between oxygen production, light intensity, culture thickness, and how much light actually reaches the algae. These variables are incredibly important when considering the implementation of algae based carbon sequestration systems in enclosed areas, where these factors can vary. 
This experiment is designed to be virtual in nature, strengthening the students' skills of data analysis and interpretation. This is done by teaching students the different impact that variables can have on the oxygen production rate of a culture of algae. Once given a set of equations that relate light intensity, illuminated area, culture thickness, students are able to use spreadsheet programs in order to visualize the impact of each. From this experiment, students are able to see how increasing the thickness of an algae culture decreases its oxygen production, while increasing light intensity increases the amount of oxygen production.

\subsection{Coagulation and Flocculation in Jar Tests}

The use of coagulation/flocculation process is incredibly important in water treatment due to the existence of nonsettleable solids. These particles are too small to be removed through conventional means such as sedimentation and filtration. Typically this is due to the small particles having an electric charge, preventing them from colliding and settling with other particles. However, by adding chemical agents to the water being treated, destabilization and aggregation can be enhanced, allowing the formation of larger particles called flocs, which can be removed through the uses of the more conventional methods. The most common chemical agent, or coagulant added in water treatment are the trivalent salts of iron and aluminum. In this experiment, students will use jar tests in order to calculate the optimum dosage of a coagulant in order to properly filter algae out of a sample. One of main benefits of this experiment is that it allows students to visualize the changes they are causing in their samples.

The reason students will be using a sample of water containing algae is to teach how algae can be used in tertiary stages of water treatment processes. The primary and secondary treatment processes of wastewater are used order to eliminate the easily settled materials and to oxidize material present in wastewater, as we mentioned earlier. The water, once treated through these methods is clear and apparently clean, however it can potentially contain inorganic nitrogen and phosphorus, which need to be removed from drinking water as they can cause health issues ${ }^{7}$. Algae offers a potential solution because it uses these inorganic chemicals for its growth. Once used to remove these chemicals though, the algae itself needs to be removed from the water through the use of coagulation/flocculation processes.

This experiment, therefore, offers a hands on approach to teaching students the importance of water treatment, and the potential use of algae in order to remove toxins from water that cannot be seen by the human eye. Additionally, this experiment can be used to introduce students to the potential uses of algae in underdeveloped countries for the purpose of water treatment.

\subsection{Microtox}

As algae grows naturally in freshwater, it can be undesirable in ponds and pools, requiring removal via algaecides that can be harmful to more than just the algae. Stormwater can cause overflow in these ponds and pools, which can carry harmful chemicals from their designated place of use to natural streams, ponds, lakes, etc. A Microtox 500 Analyzer from Modern Water is used to test exactly how harmful common algaecides can be. This machine uses the bioluminescence of cryogenically frozen bacteria to show toxicity of aqueous substances by taking a baseline bioluminescence reading of the bacteria, then another reading when the substance is introduced to the bacteria. These readings can determine the percent removal of the bacteria, or the fraction of the bacteria that the substance has killed. This experiment is intended for a freshman clinic setting where students can test different algaecides and determine the lethal 
dose of each. Results teach students how these chemicals can influence ecosystems past their intended use.

\subsection{Other Experiments}

The development of algae based materials such as surfboards, use of algae in medicine and the production of electricity using algae are currently being developed. A group of faculty and students are also developing games focusing on algae growth and other uses for K-12 educators and students.

\subsection{Communication, Data Analyses and Interpretation Skills}

The project also integrates technical writing (reports, memorandums, and letters of transmittal) and presentation skills. Students also learn to use Excel/Matlab for data analyses, plotting and statistical methods.

\subsection{Ethics, Social and Environmental Injustices}

One of highlights of the project is the inclusion of progressive humanities and qualitative social sciences. Students in teams are required to watch movies and documentaries that reflect on social/environmental injustices, breach of ethics along with gender biases in STEM fields, and social prejudices. Students also participate in debates that focus on public policy and are required to study the discussions and decisions of the Whitehouse Office of Science and Technology Policy.

\subsection{Website}

A website has been created for the Algae Grows the Future project to promote outreach and make the project's resource more widely available to educational programs. The website includes all curriculum information, lab procedure handouts, and instructional videos for the lab experiments, as well as information about partners at the Adventure Aquarium CAS and the National Science Foundation. It is intended to expand the implementation of algae based learning from a local scale to a national or even global scale. Through the website, the team can continue to update any research progress, add new experimental methods and applications, and encourage other educational programs to share how this curriculum has worked for them and make suggestions as to how to improve and add versatility to the program. For more information and to access the website, visit: http://algaegrowsthefuture.wordpress.com

\subsection{Assessment}

This project is currently being implemented in two freshman engineering courses and a middle school. Pre-surveys at the start of the project have been completed. Concept maps were also obtained. Surveys will be conducted at the end of the school year and the pre- and post- survey results will be compared to assess the impact of the project on learning not only engineering fundamentals but also the inclusion of components from the humanities.

\section{Conclusions}

In order to create a more globally conscious engineer who can focus on the problems that the world faces today, the Algae Grows the Future team has developed a curriculum that can be implemented in local K-12 Outreach programs, the STEM Academy at the local high school, as well as entry level engineering courses at the University. Drawing inspiration from the NAE 
Grand Challenges and partnering with the CAS, this project hopes to spread the idea of solving problems through engineering with a focus on the people affected while simultaneously increasing interest in the math and sciences. Furthermore, the project has focused on the implementation of algae based engineering due to the incredible versatility of algae. This has allowed the development of a curriculum that has a multitude of topics but a unity in content. Students can learn about the importance of nutrition, cosmetics, plant life cycles, sustainability, and all the branches of engineering while strengthening the skills required for scientific experimentation.

Acknowledgement: This material is based upon work supported by the National Science Foundation under DUE/IUSE Grant No. 1610164. Any opinions, findings, and conclusions or recommendations expressed in this material are those of the author(s) and do not necessarily reflect the views of the National Science Foundation. 